\title{
Prototyping, developing, and iterating a gamified survey to evaluate participatory systems modelling for youth mental health: Quality assurance pilot
}

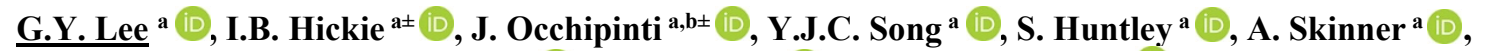 \\ K. Lawson ${ }^{a}$ iD, S.J. Hockey ${ }^{a}$ iD and L. Freebairn ${ }^{\text {a,b,c }}$ iD \\ ${ }^{a}$ Brain and Mind Centre, The University of Sydney, Australia, ${ }^{b}$ Computer Simulation \& Advanced Research \\ Technologies (CSART), Sydney, Australia, ${ }^{c}$ Research School of Population Health, The Australian National \\ University, Australian Capital Territory \\ ${ }^{ \pm}$Equal contributions \\ Email: grace.lee@sydney.edu.au
}

\begin{abstract}
Gamification is rapidly gaining popularity in research, including in participatory modelling programs. To facilitate stakeholder engagement and learning, gamification is utilised to communicate complex systems challenges at all stages of participatory modelling. However, this has excluded evaluation processes. We argue that evaluation of participatory modelling is important for refining and improving participatory processes and describe the potential for gamification to provide a more appealing platform for participants to engage in evaluation activities, eliciting richer data. This paper describes a quality assurance pilot to prototype, develop, and iterate online surveys - specifically its gamified activities - in the context of evaluating a national youth mental health participatory systems modelling program. Usability testing of the gamified surveys will be undertaken with diverse participants, which includes a rigorous process to reflect user feedback into functional improvements of the gamified activities. Potential benefits of gamification, such as participant empowerment and depth (of stakeholder learning, data, etc), are also explored. Though described in the context of an evaluation study for a national youth mental health participatory systems modelling program, this paper encourages researchers across disciplines to flexibly adapt and consider the benefits of gamification in participatory modelling including evaluation.
\end{abstract}

Keywords: Participatory modelling, gamification, evaluation, online surveys, youth mental health 


\section{INTRODUCTION}

The ubiquity of technology - including Internet access and hardware ownership - has led to significant developments in participatory modelling. In addition to software interfaces allowing the model development process to be more accessible to diverse non-technical stakeholders, technological advances have facilitated the incorporation of gamification in the participatory modelling process to improve stakeholder learning, communication, and engagement (Bakhanova et al., 2020). Though games have had a perpetual presence in human culture across history and age groups, gamification is a relatively new phenomenon with its first documented use in a 2008 blog post on social gaming (Terrill, 2008). Gamification is commonly defined as the "use of game design elements in non-game [non-entertainment] contexts" (Deterding et al., 2011). More recently a new definition has emerged whereby the goal of gamification is emphasised - "a process of enhancing a service with affordances for gameful experiences in order to support users' overall value creation" - as opposed to focusing solely on its methods as previous definitions have considered (Huotari \& Hamari, 2017).

Bakhanova et al. (2020) argue that the incorporation of gamification can benefit all stages of participatory modelling which include i) participation with stakeholders, ii) conceptual model development, iii) quantitative model development, iv) simulating solutions, and v) dissemination of results and systems learning (Voinov \& Bousquet, 2010). However, there is a recognised gap in the evaluation of participatory modelling as they are often conducted without a rigorous methodological approach, or in most instances, not conducted at all (Jordan et al., 2018). Through innovative design and implementation, evaluation not only acts as an assessment of participatory modelling, but can also facilitate improvement of both the social and technical elements of the process. For example, by revealing and addressing problems through the evaluation process, stakeholder learning and confidence in the systems model can be fostered (Hamilton et al., 2019), improving decision making capabilities at the local, state, and national government levels to manage complex issues.

Despite its recognised benefits, evaluations of participatory modelling are challenging (Falconi et al., 2017) and are generally constrained by factors including participant disengagement, program budget, resourcing, and time (Hamilton et al., 2019). Therefore, this paper investigates how gamification can be incorporated into participatory modelling evaluation to mitigate some of these challenges. Specifically, the gamification of online surveys is described in the context of a national youth mental health participatory systems modelling research program.

\section{BACKGROUND}

Mental health is a major public health concern globally that is currently exacerbated by extrinsic factors, including the pandemic, natural disasters, and recessions (The Lancet Public Health, 2020). Young people aged $12-25$ are most vulnerable to the negative impacts of mental illness with $75 \%$ of adult disorders manifesting before 25 years of age and $50 \%$ of young people experiencing first onset before 15 years of age (Hickie, 2011). Despite the recognition that many adolescent mental disorders have lifelong implications, young people in Australia continue to experience high rates of disability with the lowest rates of access to appropriate clinical care (Australian Institute of Health and Welfare, 2020). The mental health system in Australia is fragmented and overstretched, with inadequate evidence-informed decision making, evaluation, and accountability to support improvement of system performance and population mental health outcomes (Hickie, 2011). A lack of coherence in government investments in mental health at local, state, and national levels has also been attributed to poor consensus amongst the 'experts' (Grace et al., 2017; Hickie, 2011). As a result, investments in mental health over many decades continuously fail to meet the needs of young people.

Participatory modelling in youth mental health has the potential to not only improve decision making and consensus for action, but it also provides the opportunity for diverse stakeholders - including young people with lived (or living) experience of mental illness - to contribute to the modelling process (Occhipinti et al., 2021). A team of multidisciplinary researchers at The University of Sydney's Brain and Mind Centre has been leading the development of system dynamics models in youth mental health through a participatory approach, laying a robust foundation for implementation of the Right care, first time, where you live research Program (hereafter referred to as the Program). This Program aims to improve evidence-informed decision making in policy and planning, providing a tangible solution to tackle persistent challenges in complex youth mental health systems (The University of Sydney, 2020). System dynamics models will be co-designed with eight diverse sites located across Australia (including urban, outer-urban, regional, and rural-remote communities) between 2022 and 2025. A comprehensive multi-scale evaluation framework that measures the feasibility, value, impact, and sustainability of participatory systems modelling will be deployed through novel methods including participatory action research and the gamification of online surveys. The development of this framework, as well as the Program's evaluation study protocol of the participatory modelling process have 
been submitted for publication elsewhere. This paper describes a quality assurance pilot to prototype, develop, and iterate an online survey platform - specifically its gamified activities - through a cycle of usability testing with diverse participants. The potential benefits and challenges of incorporating gamification in participatory modelling will also be explored, highlighting examples from the Program's evaluation approach.

\section{OBJECTIVES}

1. To develop a prototype platform that supports gamification of online surveys and undertake an iterative usability testing process.

2. To explore the benefits and challenges of implementing gamification, in the context of a national youth mental health participatory systems modelling evaluation study.

\section{METHODS}

A series of participatory modelling workshops will be conducted from 2022 to 2025, with system dynamics models developed for two sites per year. Protocols that describe the Program's participatory modelling approach, evaluation process, as well as the blueprint to develop the technical system dynamics models have been submitted for publication elsewhere. In summary, three workshops will be conducted per site, with evaluation points ex ante and ex post (i.e., before workshop one, first follow-up after the third workshop, second follow-up 6-months after workshop three). The online surveys will incorporate gamification and will be implemented to collect evaluation data. Baseline data collection will commence in the first two Program sites from November 2021. This will be preceded by development and usability testing of the online survey gamification platform. Figure 1 depicts the timeline to prototype, develop, and iterate the gamified activities, aligned to the rest of the research Program.

For clarity, participants are referred to as individuals who agree to partake in the usability testing (quality assurance) interviews, and stakeholders are referred to as individuals who are invited to the participatory modelling workshops of the Program.

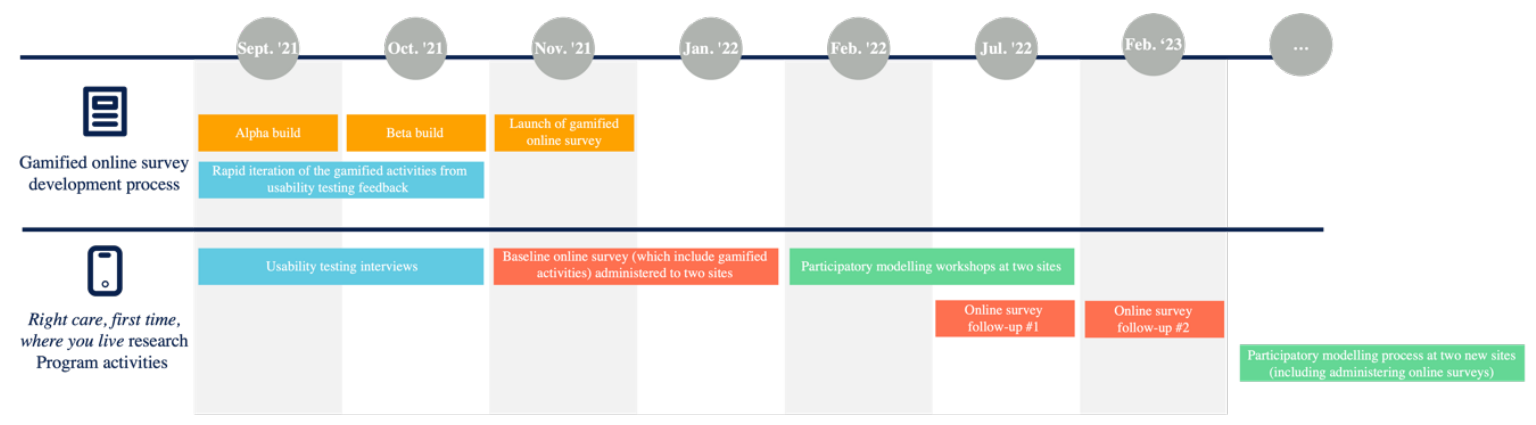

Figure 1. Timeline to prototype, develop, and iterate online surveys (including its gamified activities). The surveys aim to evaluate the participatory modelling process of the Program.

\subsection{Prototyping and developing gamified online surveys}

Baseline and follow-up surveys will include gamified activities. In addition to standard survey questions (e.g., multiple choice), three activities will be gamified including a social network analysis, patient journey mapping, and youth mental health intervention prioritising activity. The justification and description of how these activities will be gamified within the online surveys are detailed in a paper submitted for publication elsewhere. For example, questions pertaining to social connections - that will enable social network analysis - will be gamified to measure if the participatory modelling process contributed to any change in interactions between stakeholders (or collaborations between organisations) across each participating local youth mental health system. Figure 2 provides an example question from the first version prototype of the social network analysis activity, which has incorporated gaming elements such as avatars (e.g., characters to represent the user and/or fictional personas to customise the user experience), rules of play (e.g., setting parameters of how users can engage and complete each activity), and a task bar (e.g., to reward users for completing each step of the activity).

The gamified survey will be developed and hosted on the Cogniss platform (Cogniss, n.d.). The authors (GYL, IH, JO, YJCS, LF) will work with the developers of the platform to prototype and develop the gamified activities, including input on design and functional requirements. The alpha and the beta versions of the gamified surveys will be completed by September and October 2021, respectively. 


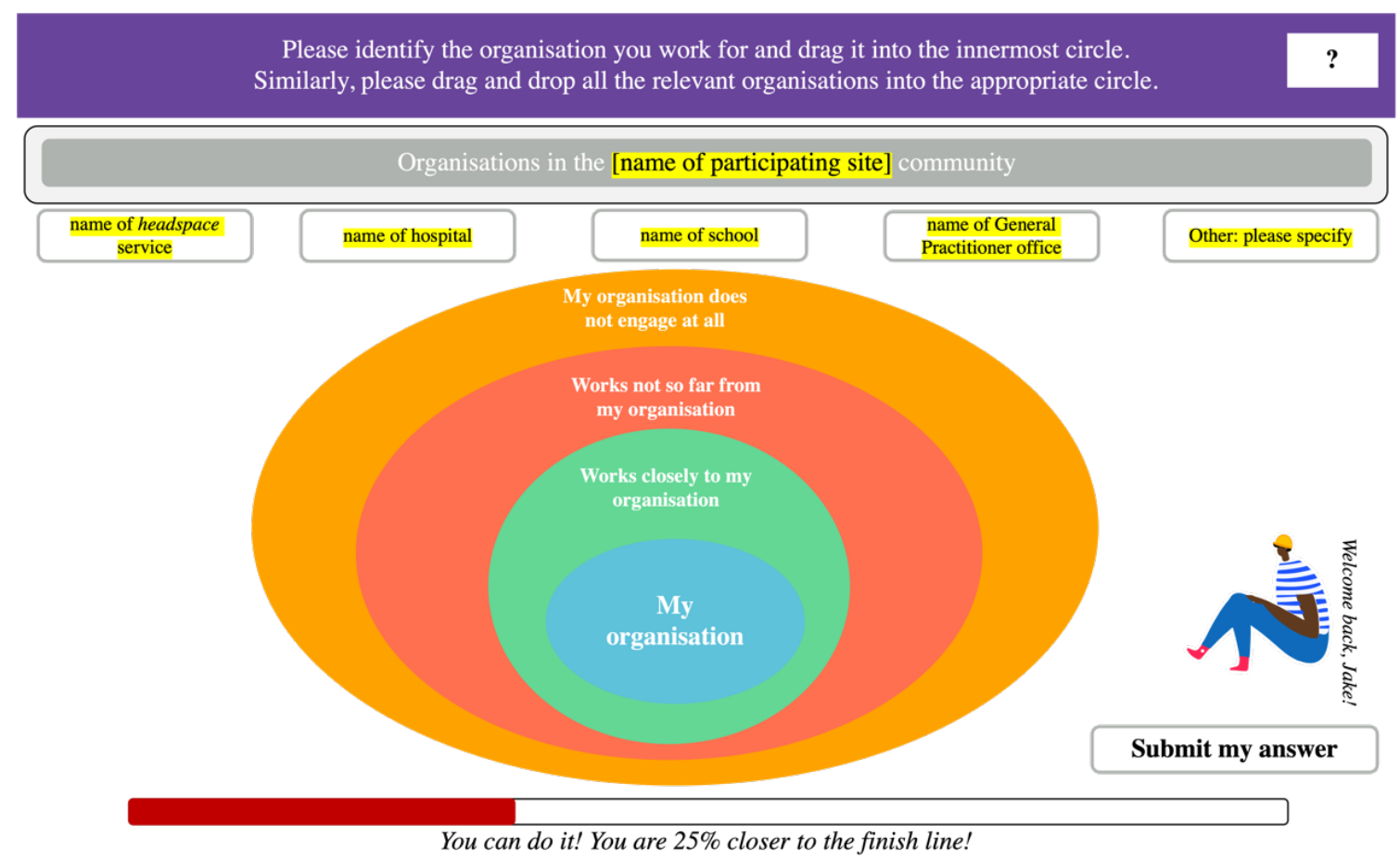

Figure 2. First version protype of a gamified online survey question that aims to understand connections between stakeholders operating across the youth mental health system; specifically, to understand how the participatory systems modelling process may have changed (e.g., improved, made worse) social network structures.

\subsection{Usability testing interviews}

Usability testing is a critical process to facilitate successful implementation of gamified applications (Elling et al., 2012). Usability testing (quality assurance) interviews will be conducted with participants that best represent stakeholders who will be invited to the participatory modelling workshops. Workshop stakeholders will include, but are not limited to, young people with lived (or living) experience of mental illness, health professionals such as psychologists, other community representatives such as teachers, and local decision makers such as staff from commissioning agencies. There will also be varying degrees of diversity of stakeholders across participating sites of the Program, such as but not limited to, cultural and educational backgrounds, as well as geography. For example, whilst some staff at a youth mental health service in a large urban city may be required to hold tertiary qualifications, the same expectation may not be required in similar service settings in rural-remote regions. Usability testing will thus be conducted with diverse participants. Importantly, participants of the usability testing interviews will not overlap with stakeholders attending the participatory modelling workshops to minimise bias in the Program evaluation.

The online surveys have already been subject to cognitive testing. Usability testing is distinct from cognitive testing as the focus will be on the ease of use and acceptance of the gamified activities, as opposed to elements of the question itself (e.g., readability). Semi-structured usability testing interviews will be conducted either in-person or online by the author (GYL) during the alpha and beta build to identify both software malfunctions (e.g., glitches) as well as the usability of the online survey itself (e.g., how long it takes for participants to complete tasks, how the gamified activities can be improved, etc). A semi-structured interview guide has been developed (Table 1), which follows a concurrent think-aloud approach whereby participants will be asked to verbalise their thoughts whilst navigating the online activities (Elling et al., 2012). Participants may also be asked to complete predetermined tasks, such as creating a user account, creating an avatar, and/or completing a specific gamified activity. In addition to what participants express verbally, the way they speak, such as their tone of voice and body language, will be observed.

Table 1. Semi-structured usability testing interview guide.

\begin{tabular}{|c|c|}
\hline $\begin{array}{c}\text { Interview } \\
\text { section }\end{array}$ & Facilitator guide / Suggested questions \\
\hline
\end{tabular}




\begin{tabular}{|c|c|c|}
\hline $\begin{array}{l}\text { Welcome and } \\
\text { introducing the } \\
\text { purpose of the } \\
\text { interview }\end{array}$ & & $\begin{array}{l}\text { Introduce participant to the aim of the interview. } \\
\text { Introduce the task to the participant (e.g., independently navigate the gamified activities to the best of their } \\
\text { ability whilst thinking aloud). } \\
\text { Introduce the role of the interviewer (e.g., to observe, listen/write notes, ask follow-up questions to clarify } \\
\text { participant's feedback). } \\
\text { Give the participant the opportunity to ask questions before commencing the 'usability testing process'. }\end{array}$ \\
\hline $\begin{array}{l}\text { Usability } \\
\text { testing process }\end{array}$ & & $\begin{array}{l}\text { If the interview is conducted in-person: The interviewer should allow the participant to have full control over } \\
\text { the online survey via computer hardware of their choice (e.g., laptop, mobile phone, tablet). } \\
\text { If the interview is conducted online: The interviewer should share their screen of the online survey and allow } \\
\text { the participant to have mouse control over the screen. } \\
\text { Example probing questions of the interview can include (but not limited to): } \\
\text { O What do you think the screen you are looking at is for? } \\
\text { What do you think the activity is asking you to do? } \\
\text { O What do you think is clickable on this page? Is anything missing? } \\
\text { O What do you think would you expect to happen if you clicked on [element]? } \\
\text { What would you expect to see on this page? Is anything missing? } \\
\text { O How would you complete [action/task]? Is it easy to complete? } \\
\text { If the participant is asked to complete a specific task, example probing questions can include (but not limited } \\
\text { to): } \\
\text { Is there anything you are unsure about? } \\
\text { After going through the [task], do you have any concerns? Why? } \\
\text { After going through the [task], is there anything you would change? Anything else? } \\
\text { What sort of trouble do you think other people may have when completing the [task]? Why? How } \\
\text { can we improve so that we can prevent this problem? }\end{array}$ \\
\hline Conclusion & & $\begin{array}{l}\text { Example concluding questions of the interview can include (but not limited to): } \\
\text { O Would you recommend the online survey (and its gamified activities) to anyone? Which activities? } \\
\text { Who and why? } \\
\text { Which activities did you think required more work? How can we improve? } \\
\text { What do you think are some benefits of the activities? What about challenges? } \\
\text { Confirm whether the participant has any further feedback/questions before concluding the interview. }\end{array}$ \\
\hline
\end{tabular}

\subsection{Iteration of gamified online surveys}

User feedback from the quality assurance interviews will be iteratively embedded into the development of the gamified surveys. Specifically, the author (GYL) will review user feedback and will provide information to the Cogniss platform developers on a weekly basis. To ensure rigour in the iteration process, user feedback will be organised into themes in accordance with the recommended approach described by Elling et al. (2012). User feedback will be organised to the following classification schemes: reading (e.g., identifying where participant's regurgitated words when reading instructions to complete activities or tasks), procedure (e.g., participant's course of actions in engaging with the activities or completing tasks), observation (e.g., participant's comments on the activities or tasks), and explanation (e.g., participant's justification of their behaviour). Feedback from the interviews will be iteratively incorporated to ensure that gamified surveys will be intuitive for all users to engage and complete. Baseline surveys will be administered to the first two Program sites in November 2021.

\section{DISCUSSION}

Gamification can be used as a supplementary approach to improve all stages of participatory modelling (Bakhanova et al., 2020); however, this has excluded evaluation processes. We argue that evaluation is important for refining and improving participatory approaches, and gamification can be a powerful method to measure the feasibility, value, impact, and sustainability of participatory modelling. Quality data can also be collected via gamified surveys by encouraging positive respondent behaviour, reducing evaluation bias (Harms et al., 2015). Some of the reported benefits of gamification in participatory modelling include stakeholder engagement, enjoyment, and motivation (Bakhanova et al., 2020). We identify two additional benefits of gamification in participatory modelling - depth and empowerment - and explain these below drawing on the Program's evaluation processes.

The incorporation of gamification in participatory modelling can facilitate depth, for instance, in stakeholder learning about the problem being studied through modelling (Mochizuki et al., 2021). In the context of the Program's evaluation processes, the gamification of online surveys allows questions to be asked in an interactive manner that can simultaneously address multiple evaluation objectives and reduce the burden on the research participant. Additionally, gamifying online surveys provide both breadth (allowing individuals to complete a quick survey at the comfort of their own hardware device of choice), as well as depth (capturing 
more detailed answers from individuals followed up by various probing questions determined by sophisticated skip and display logics that would not be possible with standard data collection tools and platforms).

Gamification can also empower individuals, particularly more vulnerable groups to gain more autonomy (van der Lubbe et al., 2021). Incorporating gamification in participatory modelling can empower stakeholders to share their experiences and knowledge, potentially leading to identification of gaps and/or solutions in the system. Using the Program's evaluation processes as an example, the gamification of the patient journey mapping activity within the online surveys gives participants control over sharing how they would navigate their local youth mental health system, while still providing useful insights to evaluators (e.g., to measure changes in participants' understanding regarding the strengths and challenges of the system as a result of the participatory modelling process). As part of the gamified activity, stakeholders of the participatory modelling workshops will be presented with a case study (or will be able to optionally share their own help-seeking experience) to understand how young people navigate services and organisations in their community to receive mental health care. This not only enables stakeholders to have control over sharing their personal experiences and knowledge, but it also allows all individuals to be recognised as experts in their experience.

Challenges in gamification are largely attributable to the paucity in research evidence and practical case examples (Bakhanova et al., 2020). This paper contributes new knowledge by describing a quality assurance pilot whereby gamified online surveys will be iteratively developed and tested. As the Program is underpinned by participatory action research (PAR), the gamified surveys will be assessed in an ongoing way. PAR is a research approach that challenges the traditional researcher-participant roles, recognising that research stakeholders contribute their expertise in their experience (such as lived mental illness). PAR also enables an ongoing cycle of reflection, action, and observation throughout the entire participatory modelling process; prompting formal check-in points to identify issues and implement solutions. Thus, PAR will allow the gamified surveys - including game design elements - to be assessed throughout the entirety of the Program to ensure that they are progressively improved to support stakeholder engagement, empowerment, enjoyment, motivation, and provide the depth or richness of information needed to undertake robust evaluation.

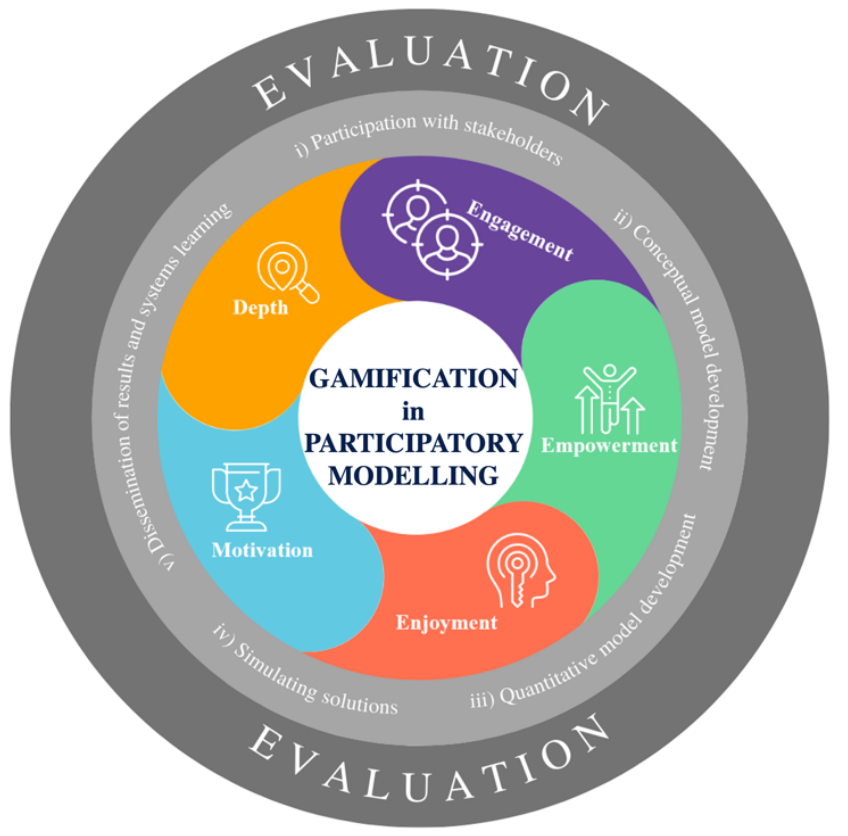

Figure 3. Potential benefits of gamification in participatory modelling, including evaluation.

\section{CONCLUSION}

As participatory modelling is more widely adopted in diverse fields to address complex issues, including the current youth mental health crisis, it is necessary to conduct comprehensive evaluations. Gamification offers promise in participatory modelling, including evaluation. This paper describes a quality assurance pilot to prototype, develop, and iterate gamified surveys to support evaluation of a national youth mental health participatory systems modelling program. This research will use a PAR approach to monitor how the incorporation of gamification in the evaluation process can mitigate challenges including long-term stakeholder 
Lee et al., Gamifying an online survey to evaluate participatory systems modelling for youth mental health

engagement, as well as conducting robust evaluations in the context of limited time, resourcing, and budget leading to important enhancements of the social and technical elements of the participatory modelling process.

\section{ACKNOWLEDGMENTS}

This research is being conducted under the Brain and Mind Centre's Right care, first time, where you live Program, enabled by a \$12.8 AUD million partnership with BHP Foundation. The Program will develop infrastructure to support decisions relating to advanced mental health, and guide investments and actions to foster the mental health and wellbeing of young people in their communities.

\section{REFERENCES}

Australian Institute of Health and Welfare, 2020. Health of young people. Canberra: AIHW, Viewed 07 August 2021, https://www.aihw.gov.au/reports/australias-health/health-of-young-people

Bakhanova E., Garcia J.A., Raffe W.L., Voinov A., 2020. Targeting social learning and engagement: what serious games and gamification can offer to participatory modelling. Environmental Modelling \& Software, $134,104846$.

Cogniss, n.d. Our Story. Viewed 09 August 2021, https://www.cogniss.com/about

Deterding S., Khaled R., Nacke L.E., Dixon D., 2011. Gamification: towards a definition. Paper presented at ACM CHI Conference on Human Factors in Computing Systems, Vancouver, Canada, May 7-12.

Elling, E., Lentz, L., de Jong, M., 2012. Combining concurrent think-aloud protocols and eye-tracking observations: An analysis of verbalizations. IEEE Transactions on Professional Communication, 53, 3, 206220.

Falconi, S.M., Palmer, R.N., 2017. An interdisciplinary framework for participatory modeling design and evaluation - What makes models effective participatory decision tools? Water Resources Research, 53(2), 1625-1645.

Grace, F.C., Meurk, C.S., Head, B.W., Hall, W.D., Harris, M.G., Whiteford, H.A., 2017. An analysis of policy success and failure in formal evaluations of Australia's national mental health strategy (1992-2012). BMC Health Services Research, 17, 374.

Hamilton, S.H., Fu, B., Guillaume, J.H.A., Badham, J., Elsawah, S., Gober, P., Hunt, R.J., Iwanaga, T., Jakeman, A.J., Ames, D.P., Curtis, A., Hill, M.C., Pierce, S.A., Zare, F., 2019. A framework for characterising and evaluating the effectiveness of environmental modelling. Environmental Modelling and Software, 118, 83-98.

Harms, J., Biegler, S., Wimmer, C., Kappel, K., Grechenig, T., 2015. Gamification of Online Surveys: Design Process, Case Study, and Evaluation. Paper presented at IFIP Conference on Human-Computer Interaction, Bamberg, Germany, September 14-18.

Hickie, IB., 2011. Youth mental health: we know where we are and we can now say where we need to go next. Early Intervention in Psychiatry, 5, 63-69.

Huotari, K., Hamari, J., 2017. A definition for gamification: anchoring gamification in the service marketing literature. Electron Markets, 27, 21-31.

Jordan, R., Gray, S., Zellner, M., Glynn, P. D., Voinov, A., Hedelin, B., et al., 2018. Twelve questions for the participatory modeling community. Earth's Future, 6, 1046- 1057.

Mochizuki, J., Magnuszewski, P., Pajak, M., Krolikowska, K., Jarzabek, L., Kulakowska, M., 2021. Simulation games as a catalyst for social learning: The case of the water-food-energy nexus game. Global Environmental Change, 66, 102204.

Occhipinti, J.A., Skinner, A., Iorfino, F., Lawson, K., Sturgess, J., Burges, W., Davenport, T., Hudson, D., Hickie, IB., 2021. Reducing youth suicide: systems modelling and simulation to guide targeted investments across the determinants. BMC Medicine, 19, 61.

Terrill, B., 2008. My coverage of lobby [sic] of the social gaming summit. Viewed 09 August 2021, http://www.bretterrill.com/2008/06/my-coverage-of-lobby-of-social-gaming.html

The Lancet Public Health, 2020. COVID-19: from a PHEIC to a public mental health crisis? Lancet Public Health, 5(8), e414.

The University of Sydney, 2020. Right care, first time, where you live. Sydney: USYD, Viewed 07 August 2021, https://www.sydney.edu.au/brain-mind/our-research/youth-mental-health-and-technology/right-carefirst-time-where-you-live-program.html

van der Lubbe, L.M., Gerritsen, C., Klein, M.C.A., Hindriks, K.V., 2021. Empowering vulnerable target groups with serious games and gamification. Entertainment Computing, 38, 100402.

Voinov, A., Bousquet, F., 2010. Modelling with stakeholders. Environmental Modelling \& Software, 25(11), $1268-1281$. 